\title{
The pharmacoeconomics of the state-of-the-art drug treatments for asthma: a systematic review
}

\author{
Francesco Menzella, ${ }^{1}$ Carla Galeone, ${ }^{1}$ Giulia Ghidoni, ${ }^{1}$ Patrizia Ruggiero, ${ }^{1}$ Maria D' Amato, ${ }^{2}$ Matteo Fontana, ${ }^{1}$ \\ Nicola Facciolongo ${ }^{1}$ \\ ${ }^{1}$ Pneumology Unit, Arcispedale Santa Maria Nuova, Azienda USL di Reggio Emilia IRCCS, Reggio Emilia \\ ${ }^{2}$ Department of Pneumology, AO "Dei Colli", University of Naples Federico II, Naples, Italy
}

\begin{abstract}
Asthma is a chronic disease characterized by significant morbidities and mortality, with a large impact on socio-economic resources and a considerable burden on health-care systems. In the standard care of asthma, inhaled corticosteroids (ICS) associated with long-acting $\beta$-adrenoceptor agonists (LABA) are a reliable and often cost-effective choice, especially if based on the single inhaler therapy (SIT) strategy; however, in a subset of patients it is not possible to reach an adequate asthma control. In these cases, it is possible to resort to other pharmacologic options, including corticosteroids (OCS) or biologics. Unfortunately, OCS are associated with important side effects, whilst monoclonal antibodies (mAbs) allow excellent results, even if far more expensive. Up to now, the economic impact of asthma has not been compared with equivalent indicators in several studies. In fact, a significant heterogeneity of the cost analysis is evident in literature, for which the assessment of the real cost-effectiveness of asthma therapies is remarkably complex. To maximize the cost-effectiveness of asthma strategies, especially of biologics, attention must be paid on phenotyping and identification of predictors of response. Several studies were included, involving comparative analysis of drug treatments for asthma, comparative analysis of the costs and consequences of therapies, measurement and evaluation of direct drug costs, and the reduction of health service use. The initial research identified 389 articles, classified by titles and abstracts. A total of 311 articles were excluded as irrelevant and 78 articles were selected. Pharmacoeconomic studies on asthma therapies often report conflicting data also due to heterogeneous indicators and different populations examined. A careful evaluation of the existing literature is extremely important, because the scenario is remarkably complex, with an attempt to homogenize and interpret available data. Based on these studies, the improvement of prescriptive appropriateness and the reduction of the use of healthcare resources thanks to controller medications and to innovative therapies such as biologics partially reduce the economic burden of these treatments. A multidisciplinary stakeholder approach can also be extremely helpful in deciding between the available options and thus optimizing healthcare resources.
\end{abstract}

Key words: Asthma; cost-effectiveness; pharmacoeconomics; biologics; oral corticosteroids; treatments.

Correspondence: Francesco Menzella, Pneumology Unit, Arcispedale Santa Maria Nuova, Azienda USL di Reggio Emilia IRCCS, 42123 Reggio Emilia, Italy. E-mail: francesco.menzella@ausl.re.it

Contributions: FM, conceptualization, writing, original draft preparation, supervision; CG, GG, PR, writing, editing; MDA, writing, original draft preparation; MF, literature search, editing, writing; NCF, supervision, coordination and editing.

Conflict of interest: FM has received research grants from AstraZeneca, Novartis and Sanofi; lecture fees and advisory board fees from AstraZeneca, Boehringer Ingelheim, Chiesi, GSK, Mundipharma, Angelini, Novartis, and Sanofi. MDA has received lecture fees and advisory board fees from AstraZeneca, GSK, Sanofi. The other authors declare no conflict of interest.

Availability of data and materials: Not applicable.

Ethics approval and consent to participate: Not applicable. 


\section{Introduction}

Asthma is a chronic disease characterized by significant morbidity and mortality, with a large impact on socio-economic resources [1] and a considerable burden on health care systems.

In USA asthma is associated with approximately US Dollars (USD) 50 billion of total direct increase annual costs [2], USD 3.8 billion in lost productivity annual costs and USD 2.1 billion per year in lost productivity due to mortality [3].

Inhaled corticosteroids (ICS) in combination with long-acting $\beta$-adrenoceptor agonists (LABA) represent the most important and effective category of drugs for the treatment of asthma [4]. A subset of patients is not adequately controlled with inhalation therapies and requires other drugs, such as long-acting muscarinic antagonists (LAMA) [5], oral corticosteroids (OCS) [6] or leukotriene receptor antagonists (LTRA) [7]. Overall, less than 5\% of asthmatics remain uncontrolled despite high dose of these treatments [8] and may require biologics as an additional option. These patients are defined as "severe asthmatics" $[9,10]$.

Omalizumab was the only biologic drug available for asthma until 2015, it is an anti-immunoglobulin E antibody (anti-IgE) indicated for patients with refractory allergic asthma [11,12]. More recently, other biologics have become available, such as mepolizumab, reslizumab and benralizumab. These are target antiinterleukin-5 (anti-IL-5) or anti-IL-5 receptor drugs, indicated as add-on therapy for patients with severe eosinophilic asthma [1315]. On the contrary, dupilumab is a fully human monoclonal antibody against the $\alpha$ subunit of the interleukin (IL)-4 and IL-4/IL-13 receptor complexes, which showed efficacy the "type-2 high" (T2) phenotype [16]. Despite the large number of pharmacoeconomic studies and cost-effectiveness analyses (CEA) on asthma, there is still no standard approach to evaluate economic costs and clinical benefits of therapies, therefore it is hard for health care decision makers seeking to distribute resources.

In asthma CEA the outcome is generally defined on clinical parameters such as symptom-free days, reduction in the rate of exacerbations, etc. The preferred outcome in terms of CEA is the quality-adjusted life years (QALY). This result estimates alternative specific survival and assigns utility weights ranging from zero (death) to one (perfect health) for each year of life gained after the therapeutic intervention. Another important outcome in a CEA is the incremental cost-effectiveness ratio (ICER). The ICER is the average incremental cost associated with an additional unit of effect and is calculated by dividing the difference in costs $(\Delta \mathrm{C})$ between intervention and comparator, over the difference in effects $(\Delta \mathrm{E})[17]$.

As part of the economic evaluations, both the cost estimates and the consequences of using different therapeutic alternatives available are often included. Comparative data on the economic value of treatments would be useful in order to make correct choices of therapeutic interventions. When a treatment option is dominant (lower cost for equal benefits, or producing a higher benefit without being more expensive than its comparator), it is unequivocally cost-effective. However, if a drug generates additional benefits, it can still be considered cost-effective even in the case of increased costs. For these reasons, it is important to obtain accurate pharmacoeconomic data in terms of health resources savings and reduction of side effects due to a lower use of OCS (often as a positive consequence of a better control of asthma due to biological drugs).

The objective of this review is to establish the real cost-effectiveness of current asthma pharmacological strategies, with a specific focus on therapies based on monoclonal antibodies.

\section{Methods}

\section{Study selection and data source}

We conducted a systematic review of the English literature from January 2000 to April 2021, concerning comparative cost analyses on drug therapies available for asthma. The search was carried out on the following biomedical bibliographic databases: MEDLINE (PubMed), EMBASE, SCOPUS, the NHS Economic Evaluation Database (NHS EED). The search strategy included the following search terms: "Economics, Medical" OR "Cost-Benefit Analysis" AND "Asthma" AND "Cost-effectiveness", "Biologics costs" AND "Pharmacoeconomics asthma treatments". Studies that met the following criteria were included: (1) comparative analysis of drug treatments for asthma; (2) comparative analysis of the costs and consequences of therapies; (3) measurement and evaluation of the direct costs of drugs and reduction of the use of the health service. Studies published exclusively in abstract form were excluded. In addition, a manual search of reference lists in publications was also carried out with the purpose of identifying any relevant missing articles (including systematic reviews, dissertations, conference proceedings, opinion articles, editorials and conference abstracts).

\section{Results}

The initial search identified 389 articles, rated across titles and abstracts. A total of 311 articles were excluded as not relevant. This led to select 78 articles that were analyzed based on the inclusion and exclusion criteria described above.

It should be noted that the economic impact of asthma has not been compared with equivalent indicators in several studies. In fact, there is a significant heterogeneity of the cost analysis in the literature, for which it was not possible to perform a quantitative meta-analysis to aggregate data on pharmacoeconomic analyses and CEA. In particular, the resources used to derive health-care costs and economic assessments vary substantially between studies, as does the type of currency considered. There was no adequate homogeneity in terms of participants, interventions and the way in which the results were defined and measured, to be able to carry out a meta-analysis. As a consequence, we decided to choose a qualitative analysis.

The costs published in different currencies in the articles examined (Euro, Great British Pound, Swedish Krona, etc.) were converted into USD to make it easier to compare the economic outcomes of the various drug options. The selected articles were reviewed in accordance with Drummond's criteria and Preferred Reporting Items for Systematic Reviews and Meta-Analyses (PRISMA) guidelines [18,19] (Figure 1).

\section{Pharmacological options}

\section{ICS/LABA}

Several studies looked at the cost-benefit ratio of ICS given alone or more often in combination with LABA (Table 1) and they demonstrated the cost effectiveness of ICS in patients with persistent asthma [20-22]. Regarding the choice between different ICS, there are reports suggesting that fluticasone propionate (FP) is more cost-effective than budesonide (BUD) or flunisolide [23]. A study performed in developing countries concluded that FP may be more cost-effective than beclomethasone dipropionate (BDP) [24]. 
In inadequately controlled asthma patients despite taking ICS, adding LABA (salmeterol or formoterol) to ICS (FP, BUD or BDP) is cost-effective compared to ICS alone at the same doses [25-29] or in double doses $[27,28]$. Other studies reported that fixed combinations of ICS and LABA were more cost-effective than the same drugs in separate inhalers [29]. As regard to the choice between different LABAs, an old study did not show that LABA alone (formoterol and salmeterol) was more cost-effective than the other [30]. More recent data showed that formulations with ICS and formoterol could be more cost effective compared to combinations of ICS and salmeterol, being associated with cost savings and a superior clinical efficacy $[31,32]$. The greatest evidence of efficacy emerged when ICS and formoterol combination (BUD/formoterol is the most studied combination) was used for both single inhaler therapy (SIT) [33]. There are now numerous data confirming that the SIT strategy [33] is superior and cost-effective compared to alternative strategies, such as FP/fixed-dose salmeterol plus salbutamol or terbutaline needed as reliever therapy. A study based on a Markov model estimated the costs and health outcomes associated with therapeutic step-down in patients with controlled asthma on high-dose $\mathrm{FP} / \mathrm{S} 1000 / 100 \mu \mathrm{g}$ per day treatment who switched to FP PMDI/S $(500 / 100 \mu \mathrm{g})$ medium dose dry powder or $\mathrm{BDP} / \mathrm{F} 400 / 24 \mu \mathrm{g}$ extrafine ) [34].

In this study, the CEA showed that high FP / S versus medium dose FP / S were not cost effective options versus medium dose BDP / F (400/ $24 \mu \mathrm{g})$, with a low probability (less than 1\%) to be cost-effective, when USD 25,000 / QALY threshold is used. Consequently, maintenance therapy with high-dose 1000/100 $\mu \mathrm{g}$ daily FP/S was not cost-effective compared to extrafine $400 / 24 \mu \mathrm{g}$ $\mathrm{BDP} / \mathrm{F}$ [34].

There are some limitations: most of those who compared ICS with a combination ICS/LABA drew the efficacy data from RCTs and identified a limited number of comparators. Furthermore, many were sponsored by a pharmaceutical company or the source of funding was not clearly stated and had a short time horizon (on average less than 1 year, ranging from 12 to 24 weeks).

A very low number of asthma CEA evaluated adherence, which is known to be very low in asthma. A recent review found that within CEA, only four studies $(17.4 \%)$ had incorporated adherence in analyzes [35]. A study by Shih et al. estimated cost effectiveness of fluticasone propionate plus salmeterol administered in a single inhaler, compared with fluticasone propionate inhaled corticosteroids, non-fluticasone propionate and LTRA [36]. A study by Rodriguez-Martinez et al. showed that beclomethasone dipropionate was associated with the lowest cost, while fluticasone propionate had the highest number of QALY [24]. Beclomethasone dipropionate was the most cost-effective option when the willingness-to pay threshold was below USD 29,30/QALY. Also, in this study a comparison between budesonide twice daily versus budesonide once daily showed that a once daily dose was a dominant strategy [24]. Another study evaluated the cost-effectiveness of improving adherence, comparing the hypothetical scenarios, in which all patients fully adhered to therapies (full adherence) and the real scenario (the actual patient adherence data) [37]. The authors showed that full adherence makes the therapeutic strategy more cost-effective.

\section{Tiotropium}

Tiotropium is a drug with few pharmacoeconomic studies conducted on it in asthma (Table 1). One study evaluated tiotropium as add-on option to usual care in asthma patients showing that it was cost effective, resulting in an ICER of USD 30,38 per QALY gained [38]. A recent study evaluated the cost-effectiveness of adding tiotropium to ICS/LABA in elderly patients with severe asthma (SA) in a real-life setting [38]. The ICER was USD 83,30 /QALY, indicating that the addition of tiotropium is not a costeffective alternative. Despite this, two subgroups with poorly controlled asthma showed that adding tiotropium was a cost-effective alternative (frequent SABA users USD 5,65/QALY; frequent exacerbators USD 11,55/QALY). In conclusion, tiotropium showed a high cost-effectiveness profile only when applied to elderly patients with uncontrolled symptoms [39].

A study based on a Markov model evaluated total costs and health outcomes over a 10-year time horizon of three treatments including standard therapy (ICS + LABA), add-on therapy with tiotropium and add-on therapy with omalizumab [33]. The 10years and QALYs for standard therapy were USD 38,432 and 6.79, respectively. Corresponding values for tiotropium and omalizumab add-on therapy were USD 41,535 and 6.88 and USD 217,847 and 7.17 , respectively. The ICER of tiotropium add-on therapy compared to standard therapy and omalizumab versus tiotropium was USD 34,478/QALY and USD 593,643/QALY, respectively. Despite the greater impact on asthma control achievable with omalizumab, add-on therapy with tiotropium resulted in a viable alternative to the latter and to standard uncontrolled allergic asthma therapy with willingness to pay USD 50,000/QALY [40].

\section{LTRA}

In the same way, different studies showed that in patients with mild-to-moderate persistent asthma, ICS had lower costs and higher effectiveness compared with LTRA preparations such as montelukast (MON) or zafirlukast [41-43] (Table 1). An exception to this statement might occur in case ICS adherence cannot be achieved, in which case an economic evaluation based on administrative claims data from US commercial health plans found that LTRA may be a reasonable choice for monotherapy [44]. Other

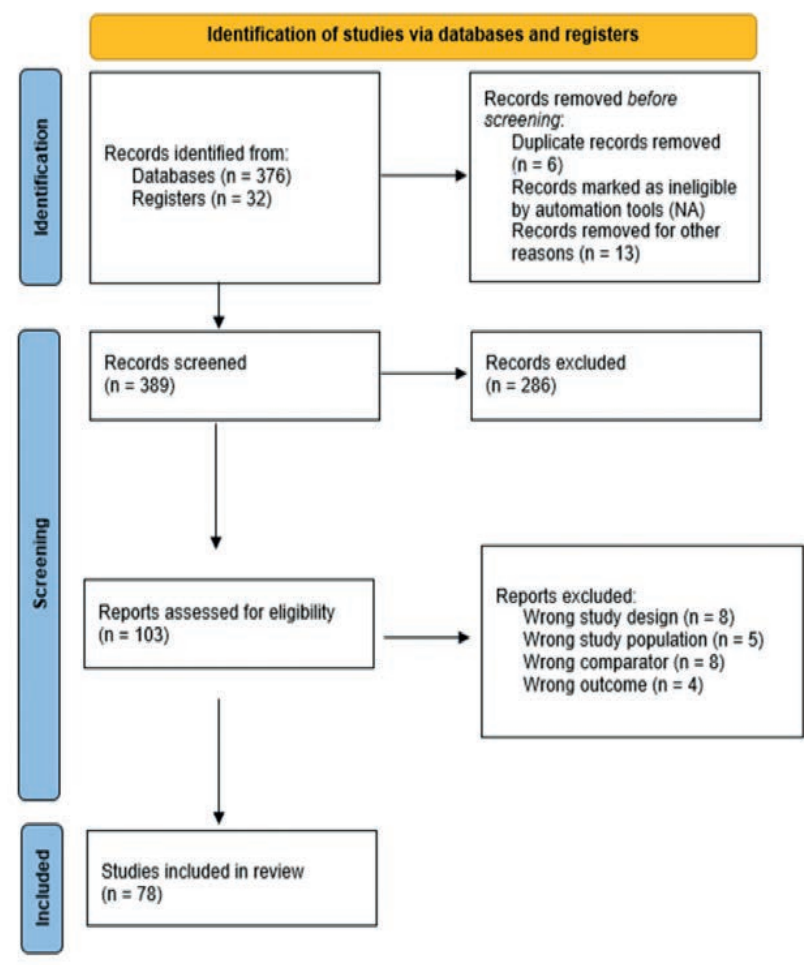

Figure 1. PRISMA flow diagram. 
studies conducted in patients with uncontrolled asthma with ICS showed that the addition of LABA such as salmeterol to ICS (e.g., FP) may be cost effective compared to adding an LTRA such as MON at similar doses to ICS [45]. Only a pragmatic trial demonstrated a slight economic advantage of LTRAs over LABAs as add-on treatment to ICS [46]. In the cost-benefit comparison between salmeterol/FP to MON as maintenance monotherapy in patients treated with Short-Acting Beta-Agonists (SABAs) alone
[47-49], SFC therapy showed a higher percentage of days without symptoms, a greater number of weeks without SABA use and a higher percentage of patients who achieved a $12 \%$ or greater increase in forced expiratory volume in $1 \mathrm{~s}\left(\mathrm{FEV}_{1}\right)$ from baseline.

Even in this case, there were methodological issues such as comparisons of unequal treatment groups or inappropriate use of leukotriene modifiers in stepwise treatment. Moreover, evidence on LTRAs has similar limitations to those described for ICS-

Table 1. Cost analysis of different drug options for asthma.

\begin{tabular}{lrl}
\hline Author & Year & Pharmacological options \\
Sullivan et al. [19] & 2003 & BUD added to SABA \\
Bruggenjurgen et al. [28]2010 & $\begin{array}{l}\text { BUD/F fixed combination compared } \\
\text { to the same drugs via separate inhalers }\end{array}$ \\
\hline Gerzeli et al. [30] & 2012 & BDP/F versus FP/ Salmeterol \\
& & \\
Miller et al. [32] & 2007 & $\begin{array}{l}\text { BUD / F in a single inhaler used as both } \\
\text { maintenance and reliever medication (SMART) } \\
\text { versus salmeterol-FP plus as needed SABA }\end{array}$ \\
\hline Willson et al. [37] & 2014 & $\begin{array}{l}\text { Tiotropium as add-on in patients uncontrolled } \\
\text { with ICS/LABA }\end{array}$
\end{tabular}

Population / outcomes

7165 subjects aged from 5 to 66 years.

The ICER was USD 15,60 per symptoms free day gained adding budesonide. Long-term treatment with BUD appears to be cost-effective in patients with mild persistent asthma of recent onset.

30 patients $18-70$ years old.

The incremental cost-effectiveness ratio was reduced by USD 11,44 per

additional day free of asthma symptoms with the fixed combination. BUD/F fixed combination is superior to BUD + F delivered via separate inhalers.

Study based on a Markov model.

$\mathrm{BDP} / \mathrm{F}$ led to a reduction of cost of USD 69.74 per patient per year, and a gain in QALY of 0.0032 .

From the Italian NHS perspective, BDP/F treatment is associated with a reduction in cost

and offers a slight increase of effectiveness in terms of weeks spent in successful control and QALYs.

2143 patients $12-84$ years old.

ICER in favor of BUD / F SMART, with an incremental difference of USD 226.

SMART proved to be more effective and less expensive than a strategy of clinician-directed titration of salmeterol-FP with salbutamol as reliever therapy.

Study based on a Markov model.

Add-on tiotropium resulted in an ICER of USD 30,20 per QALY gained.

Tiotropium was found to be cost effective when added to usual care in patients who remain uncontrolled despite treatment with high-dose ICS/LABA.

\begin{tabular}{|c|c|c|c|}
\hline Hong et al. [38] & 2020 & $\begin{array}{l}\text { Tiotropium as add-on in elderly patients with } \\
\text { severe asthma treated with ICS/LABA }\end{array}$ & $\begin{array}{l}\text { Study based on National Health Insurance claims data and on a Markov model. } \\
\text { Add-on tiotropium is cost effective only in frequent SABA users and in frequent exacerbators, } \\
\text { with ICER per QALY of USD } 5.62 \text { and USD 11.5, respectively. } \\
\text { Tiotropium shows a higher cost-effectiveness profile when applied to elderly patients } \\
\text { with uncontrolled symptoms. }\end{array}$ \\
\hline Menendez et al. [40] & 2001 & $\begin{array}{l}\text { Zafirlukast versus FP in patients } \\
\text { taking SABA alone }\end{array}$ & $\begin{array}{l}451 \text { patients, mean age } 13 \text { years. } \\
\text { Zafirlukast resulted more costly and less effective compared with FP. }\end{array}$ \\
\hline Canonica et al. [58] & 2019 & $\begin{array}{l}\text { Pharmacoeconomic analysis of OCS } \\
\text { adverse events }\end{array}$ & $\begin{array}{l}\text { Study based on a pharmacoeconomic model and epidemiologic data from the Severe Asthma } \\
\text { Network in Italy (SANI) registry. } \\
\text { Estimated total annual cost related to OCS-related adverse events of USD 286,3 million for severe } \\
\text { asthmatics incremental expenditure of about USD } 130,5 \text { million in respect with non-asthmatics } \\
\text { and USD } 88,70 \text { in respect with moderate asthma population. } \\
\text { Budget impact model results highlighted the relevant economic impact of OCS-related adverse events } \\
\text { in severe asthma patients. }\end{array}$ \\
\hline Oba et al. [60] & 2004 & Omalizumab & $\begin{array}{l}1071 \text { patients } 12-76 \text { years old. } \\
\text { Annual direct cost for patient from USD 21,000 to USD } 62,265 \text {. } \\
\text { Omalizumab could be cost saving if given to nonsmoking allergic asthmatic patients who are } \\
\text { hospitalized } 5 \text { or more times or } 20 \text { days or longer per year despite maximal asthma therapy. }\end{array}$ \\
\hline Tan et al. [63] & 2019 & Mepolizumab & $\begin{array}{l}\text { Study based on a Markov model. } \\
\text { ICER high: USD 2,400 per QALY. } \\
\text { At its current price, mepolizumab is not considered a cost-effective use of healthcare resources in } \\
\text { Singapore. Substantial price reductions for mepolizumab are required to improve its cost-effectiveness. }\end{array}$ \\
\hline Andersson et al. [64] & 2020 & Benralizumab & $\begin{array}{l}\text { Study based on a Markov model. } \\
\text { Cost-effective compared to usual care plus OCS for patients with severe eosinophilic asthma } \\
\text { who receive regular OCS treatment. } \\
\text { Benralizumab has a high probability of being cost-effective compared with SOC plus OCS for a } \\
\text { subgroup of patients with severe, eosinophilic asthma receiving regular OCS treatment. }\end{array}$ \\
\hline
\end{tabular}

ICS, Inhaled corticosteroids; ICS/LABA, Inhaled corticosteroids/ong acting beta-adrenoceptor agonists; LTRA, leukotriene receptor antagonists; OCS, oral corticosteroids; ICER, Incremental Cost-Effectiveness Ratio; QALY, Quality Adjusted Life Years; BDP, budesonide; F, formoterol; FP, fluticasone propionate; SOC, standard of care. 
LABA studies. The cost-effectiveness comparison in the SFC studies versus LTRA alone as initial maintenance therapy for persistent asthma in patients treated with SABA [48-50] has some limitations. Firstly, considering improvement in lung function relative to clinical outcomes among drugs with or without bronchodilator (such as MON) as the primary outcome may limit the validity of the results. Furthermore, the comparison between SFC and LTRA does not fully reflect the indications of guidelines, because SFC is not a first-line treatment compared to LTRA but it is used in patients with uncontrolled asthma with ICS only. In a retrospective study based on real-life data with the aim to compare asthma-related healthcare costs between patients on new prescription of FP 44 or $110 \mu \mathrm{g}$, MON 5 or $10 \mathrm{mg}$, or zafirlukast $20 \mathrm{mg}$, fluticasone propionate showed to be more effective than LTRAs [51]. Cost-savings were a consequence of the lower use of healthcare resources, combined with the lower cost of drugs present in the FP group. These results confirm the recommendation of ICS as a first-line controller for asthma over LTRAs.

\section{OCS}

OCS, due to their powerful anti-inflammatory effect, have been the most potent therapy for refractory asthma for decades. They are often prescribed intermittently to treat severe exacerbations, although a subset of patients requires prolonged or chronic therapy [52].

There is no real safe dose of OCS as they are unfortunately associated with numerous and known long-term side effects (such as arterial hypertension, osteoporosis, bone fracture, cataract, glaucoma, diabetes, respiratory infections, reduced growth rate in children and suppression of the hypothalamic-pituitary-adrenal axis), with increased mortality [53]. Recent evidence suggested that this risk is related to the duration and cumulative dose of OCS [54,55], which means that even short but repeated courses can have a significant impact on morbidity. Patients with SA and T2-high inflammation are those who best respond to corticosteroids but often require high, continuous dosages. Conversely, those with T2 low asthma often have poor responsiveness to OCS treatment. Few studies evaluated the economic impact of OCS-related adverse events in the treatment of asthma [56,57]. The estimated annual cost for asthmatic patients ranges from around USD 700 to around 6000 for the most severely ill patients [58]. A recent study based on a pharmacoeconomic model calculated the costs of adverse events from OCS in patients with SA based on epidemiological data published by Severe Asma Network Registry in Italy (SANI) [59] (Table 1). Comparing the annual costs for SA versus the nonasthmatic population, the authors indicated an incremental expenditure of approximately USD 132 million, which was reduced to approximately USD 90 million in patients with moderate asthma.

The data show that a significant portion of the large increase in SA costs compared to patients with moderate asthma is due to exposure to OCS. OCS-induced prescription costs averaged around USD 155 for patients exposed to moderate doses and USD 1,070 for those with high exposure. Consequently, $51 \%$ of the difference in clinical costs can be attributed to OCS-induced morbidity for patients with SA compared to $39 \%$ for patients with moderate asthma [60].

The advent of biological therapies for SA offered effective pharmacological options with a powerful OCS sparing effect, leading to significant reductions in the average dose of corticosteroids or complete weaning from them.

\section{Biologics}

Monoclonal antibodies (mAbs) are highly effective though far more expensive, compared to drug options discussed so far. This aspect makes pharmaco-economic analyses more complex, but crucial for clinicians and decision makers. The failure of standard care with high dose ICS/LABA and other controllers requiring continuous or frequent courses of OCSs is the main criterion to define a patient eligible for therapy with biological drugs. Nevertheless, biological drugs should be limited only to selected patients with specific phenotypes potentially responsive to individual options. Approximately, the annual direct costs of omalizumab are in the range of USD 21,000 to 62,000 per patient. One study suggested that, given the large increase in direct costs, only patients hospitalized $>$ five times or $>20$ days per year could qualify for omalizumab with a viable pharmacoeconomic basis [61]. Other data showed that omalizumab add-on therapy resulted in an improvement in QALY but with a significant increase in direct medical costs and a poor ICER [62].

Long-term clinical outcomes were not frequently evaluated in pharmacoeconomic studies and overall short-term convenience was predominantly examined. One study performed a pre-post comparison of clinical outcomes and health care consumption in a cohort of selected subjects over a 36-month time horizon. In these patients, add-on therapy with omalizumab significantly and progressively improved asthma control and Health-related quality of life (HRQoL). The symptomatic costs of medications and hospital care for these patients decreased significantly. An increase of 450 euros in total monthly costs was observed, but this increase in costs translated into an incremental cost / utility ratio of $€ 23,880$ per year of life earned, a fairly favorable and convenient figure. This study demonstrated that add-on therapy with 36 months of omalizumab persistently improved all clinical outcomes in difficult-totreat asthma patients [63]. A retrospective study based on a long follow-up (4 years) confirmed the efficacy and safety of omalizumab, highlighting that additional costs were offset by the medium and long-term savings, associated with the reduction in hospitalizations and access to emergency departments [64].

About the other biologics, few pharmacoeconomic studies are available. An analysis conducted on mepolizumab showed that ICER is very sensitive to its price, followed by the rate of exacerbations requiring hospitalization. Despite limiting the use of mepolizumab to patients with frequent exacerbations at baseline (3 in the past year) in a scenario analysis, the ICER remained high (USD 1,198 per QALY earned). Consequently, at its current price, mepolizumab was not considered cost-effective except on the condition of significant price reductions [65]. In a recent Swedish analysis, benralizumab demonstrated cost-effectiveness compared to usual care plus OCS with USD 4,404 per QALY gained, based on increases of 1.33 QALY and USD 5,868 per patient. The results of this study showed that benralizumab had a high probability of being cost-effective compared to usual care plus OCS for a subgroup of patients with severe eosinophilic asthma who received regular OCS treatment [66]. According to a Canadian pharmacoeconomic review report, comparing biologics for the treatment of severe asthma was difficult due to differences in indication and lack of head-to-head studies. Based on similar safety and efficacy, benralizumab was considered more expensive than mepolizumab and omalizumab, also because the purchase cost was higher. The price of benralizumab should be reduced by $4 \%$ to be less expensive than mepolizumab and by $7 \%$ to be cheaper than omalizumab [67]. Literature data comparing the 5 biologics available (including reslizumab and dupilumab) indicate that current prices exceed cost-effectiveness measures (Table 1). In order to improve the situation, prices would have to be reduced by at least around $60 \%$ [68]. Similarly, according to the ICER report "Biological Therapies for the Treatment of Asthma Associated with Inflammation Type 2: Value-Based Efficacy, Value and Price Benchmarks", to align costs with the additional benefits of all asthma biologics, net prices should be discounted between $50 \%$ and 
$79 \%$ [69]. Home administration is a strategy to at least partially reduce the costs associated with biological treatments. This is a relatively recent possibility, but some authors showed that it could be cost-effective compared to routine administration in clinics. The latter almost never seemed to be cost-effective [70].

\section{Conclusions}

Asthma is a large economic burden worldwide, not only for national health systems but also for patients and society, due to the loss of productivity and the increased use of healthcare resources. The great speed, in which new pharmacological options are available, especially in the biological field, does not make the task of defining the best possible therapy easy, from both clinical and economical point of views.

In the standard care of asthma, ICS-LABAs are the most effective and often cost-effective choice, especially if based on SIT strategy, but they are not functional in terms of adequate asthma control in a subset of patients. Numerous studies confirmed that high blood and sputum eosinophil counts and high fractional exhaled nitric oxide (FeNO) level can predict a good response to ICS treatments $[71,72]$. The presence of these biomarkers should always be considered when deciding which drugs to prescribe in the context of precision medicine and to optimize costs. In these cases, it is possible to resort to tiotropium and LTRAs as additional options, with good results in many patients, although generally inferior to ICS-LABAs. LTRAs should be used more selectively than it is currently done, as patients who respond best to them are preschool, female, high cotinine and urinary leukotriene E4 (LTE4) levels [73]. Most pharmacoeconomic studies about asthma therapies have several limitations and there is also a lack of headto-head studies not only among biologics, but also among asthma controller drugs that would allow a more effective comparison of results.

The use of OCSs should be reserved only for patients who are truly refractory to the previous drugs, in case of exacerbations or if there is no indication for biologics. However, it is always important to use them for the shortest period and with the lowest possible doses, especially to minimize the risk of the OCS-related complications, which are highly expensive, both from a purely economic and biological cost point of view. The latter is more difficult to quantify as it also includes intangible costs such as the significant deterioration in HRQoL. One possibility to reduce the biological impact of OCS, with consequent increase in economic costs related to side effects and worsening of HRQoL, is to identify predictors of response. Blood and sputum eosinophils and FeNO are the predictive biomarkers of favorable response to OCS and they should guide the use of these drugs to obtain better disease control and reduce its costs, whenever possible [74,75]. The only real agents capable of minimizing or solving these problems are biological drugs, which are increasingly numerous and effective but also vastly expensive.

Systematic reviews of asthma CEA normally focused primarily on assessing the monetary value of asthma treatments [76,77]. In the future, it will also be very important to better define the impact of treatment schemes and changes in trajectories (i.e. step-up and step-down) to better define the cost-effectiveness of drugs. The time required for treatment must also be carefully evaluated, in order to obtain results less influenced by possible bias. Finally, it is necessary to have a greater standardization of data on costs, times, QoL evaluation measures and the integration of data on adherence to treatments, notoriously low but not very considered in the CEA. The scenario with full adherence is the most costeffective one, but currently real-life is very different so it is essen- tial to incorporate adherence in CEA more often. Another critical point is the choice of the time horizon. The problem is evident in asthma due to the short duration of many of the clinical studies that provide data for CEA, although in a chronic disease such as asthma, the treatment often continues for many years. Long-term pragmatic studies would probably offer the best opportunity to measure the QALY benefits of asthma treatments in the context of a more correct time horizon.

Due to often different, fragmented or inefficient care systems, great variability in treatment practices, lack of transparency of prices, inadequate pharmacoeconomic costs and data to assess value, defining the real cost-effectiveness of asthma treatments, in particular of biologicals, remains a complex exercise with uncertain results. Pharmacoeconomic studies on asthma treatments showed conflicting results also because of the heterogeneity of the indicators used in CEA and pharmacoeconomic studies. Furthermore, the various treatment options have not often been cost-effective, mainly due to the high purchase prices, despite their great effectiveness. To maximize the cost-effectiveness of asthma treatment options attention must be focused on accurate phenotyping and on identification of predictors of response, especially in patients who are frequent exacerbators requiring OCS therapy.

Another crucial point in the management of asthma is the appropriateness of therapeutic strategies. This point is highlighted by the substantial increase in pharmaceutical costs, which however can be reflected in a considerable decrease in costs related to hospital admissions. The role of an adequate pharmacological strategy is still crucial in minimizing the morbidity of asthma and the corresponding socio-economic impact. A large, retrospective observational analysis on a cohort of 817 patients with different severity levels of persistent asthma confirmed these aspects [78]. In this study, the estimated total annual cost per asthmatic patient was $€$ $1,183.14$ ( $\pm € 65.79)$ in the 12 months prior to enrollment and $€$ $1,290.89$ ( $\pm € 68.74$ ) throughout the follow up. The increase was mainly due to the significantly longer duration of the pharmacologic therapies. Despite this, costs related to hospitalizations, general practitioners and rescue medications were significantly reduced thanks to improved drugs strategies.

Pharmacoeconomic studies and CEA on asthma therapies often report conflicting data also due to heterogeneous indicators and populations examined. A careful evaluation of the existing literature is extremely important, because the scenario is remarkably complex, with an attempt to homogenize and interpret available data. We have seen how asthma "controller" drugs are often costeffective, whilst the high direct costs of biologics make them less advantageous from this point of view. The improvement of prescriptive appropriateness and the reduction of the use of healthcare resources thanks to these innovative therapies partially reduce their economic burden. Regarding biologics, a price reduction is desirable to make these important therapeutic options more cost-effective. The possibility of "home administration", allowed for all biologics except reslizumab, could permit savings for health systems and patients themselves; it can also be obtained by a more extensive use of "patient support programs" (PSP). To maximize the cost-effectiveness of asthma treatment options but especially of biologics, attention must be focused on accurate phenotyping and on identification of predictors of response, especially in patients who are frequent exacerbators requiring OCS therapy. Therefore, pharmacoeconomic studies have an increased importance at the moment because they are able to provide information that can be very persuasive for policy makers and other decision makers. Systematic analysis and correct interpretation of data provide useful information on stakeholders' perceptions of the value and effectiveness of a treatment option and its alternatives, so that choices can be made appropriately. CEA can also guide prioritization when 
resources are limited, based on documented cost-effectiveness data. A multidisciplinary stakeholder approach can also be extremely helpful in achieving the best use of available therapies and optimizing healthcare resources.

\section{Abbreviations}

USD: US Dollars;

ICS: inhaled corticosteroids;

LABA: $\beta$-adrenoceptor agonists;

LAMA: long-acting muscarinic antagonists;

OCS: oral corticosteroids;

LTRA: leukotriene receptor antagonists;

ERS/ATS: European Respiratory Society/American Thoracic

Society;

anti-IL-5: anti-interleukin-5;

IL-4 interleukin-4;

CEA: cost-effectiveness analyses;

QALY: quality-adjusted life years;

ICER: incremental cost-effectiveness ratio;

PRISMA: Preferred Reporting Items for Systematic Reviews and

Meta-Analyses);

FP: fluticasone propionate;

BUD: budesonide;

BDP: beclomethasone dipropionate;

SIT: single inhaler therapy;

MON: montelukast;

$\mathrm{FEV}_{1:}$ forced expiratory volume in $1 \mathrm{~s}$;

mAbs: monoclonal antibodies;

HRQoL: health-related quality of life;

FeNO: fractional exhaled nitric oxide;

PSP: patient support programs.

\section{References}

1. Pennington E, Yaqoob ZJ, Al-Kindi SG, Zein J. Trends in asthma mortality in the United States: 1999 to 2015. Am J Respir Crit Care Med 2019;199:1575-7.

2. Bahadori K, Doyle-Waters MM, Marra C, Lynd L, Alasaly K, Swiston J, et al. Economic burden of asthma: a systematic review. BMC Pulm Med 2009;9:24.

3. Reddel HK, Bateman ED, Becker A, Boulet LP, Cruz AA, Drazen JM, et al. A summary of the new GINA strategy: a roadmap to asthma control. Eur Respir J 2015;46:622-39.

4. Greenstone IR, Ni Chroinin MN, Masse V, Danish A, Magdalinos H, Zhang X, et al. Combination of inhaled longacting beta2-agonists and inhaled steroids versus higher dose of inhaled steroids in children and adults with persistent asthma. Cochrane Database Syst Rev 2005;(4):CD005533.

5. Befekadu E, Onofrei C, Colice GL. Tiotropium in asthma: a systematic review. J Asthma Allergy 2014;7:11-21.

6. Bleecker ER, Menzies-Gow AN, Price DB, Price DB, Bourdin A, Sweet S, et al. Systematic literature review of systemic corticosteroid use for asthma management. Am J Respir Crit Care Med 2020;201:276-93.

7. Peters SP. Leukotriene receptor antagonists in asthma therapy. J Allergy Clin Immunol 2003;111:S62-70.

8. Hekking PP, Wener RR, Amelink M, Zwinderman AH, Bouvy ML, Bel EH. The prevalence of severe refractory asthma. J Allergy Clin Immunol 2015;135:896-902.

9. Holguin F, Cardet JC, Chung KF, Diver S, Ferreira DS, Fitzpatrick A, et al. Management of severe asthma: a European
Respiratory Society/American Thoracic Society guideline. Eur Respir J 2020;55:1900588.

10. Global Initiative for Asthma. GINA Report. Accessed: 19 May 2021. Available from: https:/ginasthma.org/gina-reports/

11. Darveaux J, Busse WW. Biologics in asthma-the next step toward personalized treatment. J Allergy Clin Immunol Pract 2015;3:152-60.

12. Kumar C, Zito PM. Omalizumab. Treasure Island, StatPearls Publishing; 2021.

13. Menzella F, Lusuardi M, Galeone C, Facciolongo N, Zucchi L. The clinical profile of benralizumab in the management of severe eosinophilic asthma. Ther Adv Respir Dis 2016;10:53448.

14. Menzella F, Lusuardi M, Galeone C, Taddei S, Facciolongo N, Zucchi L. Mepolizumab for severe refractory eosinophilic asthma: evidence to date and clinical potential. Ther Adv Chronic Dis 2016;7:260-77.

15. Walsh GM. Reslizumab in the treatment of severe eosinophilic asthma: an update. Immunotherapy 2018;10:695-8.

16. Deeks ED. Dupilumab: A review in moderate to severe asthma. Drugs 2019;79:1885-95.

17. van Boven JFM, van de Hei SJ, Sadatsafavi M. Making sense of cost-effectiveness analyses in respiratory medicine: a practical guide for non-health economists. Eur Respir J 2019;53:1801816.

18. Drummond M, Brandt A, Luce B, Rovira J. Standardizing methodologies for economic evaluation in health care: practice, problems, and potential. Int J Technol Assess Health Care 1993;9:26-36.

19. Page MJ, McKenzie JE, Bossuyt PM, Boutron I, Hoffmann TC, Mulrow CD, et al. The PRISMA 2020 statement: an updated guideline for reporting systematic reviews. BMJ 2021;372:n71.

20. Sullivan SD, Buxton M, Andersson LF, Lamm CJ, Liljas B, Chen YZ, et al. Cost-effectiveness analysis of early intervention with budesonide in mild persistent asthma. J Allergy Clin Immunol 2003;112:1229-36.

21. Palmqvist M, Price MJ, Sondhi S. Cost-effectiveness analysis of salmeterol/fluticasone propionate $50 / 250 \mathrm{lg}$ vs fluticasone propionate $250 \mathrm{lg}$ in adults and adolescents with asthma IV: results. Pharmacoeconomics 1999;16:S23.

22. Johansson G, Price MJ, Sondhi S. Cost-effectiveness analysis of salmeterol/fluticasone propionate $50 / 100 \mathrm{lg}$ vs fluticasone propionate $100 \mathrm{lg}$ in adults and adolescents with asthma III: results. Pharmacoeconomics 1999;16:S15.

23. Mukhopadhyay A, Waked M, Gogtay J, Gaur V. Comparing the efficacy and safety of formoterol/budesonide pMDI versus its mono-components and other LABA/ICS in patients with asthma. Respir Med 2020;170:106055.

24. Rodríguez-Martínez CE, Sossa-Briceño MP, Castro-Rodriguez JA. Cost-utility analysis of the inhaled steroids available in a developing country for the management of pediatric patients with persistent asthma. J Asthma 2013;50:410-8.

25. Andersson F, Stahl E, Barnes PJ, Löfdahl CG, O'Byrne PM, Pauwels RA, et al. Adding formoterol to budesonide in moderate asthma-health economic results from the FACET study. Respir Med 2001;95:505-12.

26. Doull I, Price D, Thomas M, Hawkins N, Stamuli E, Tabberer $\mathrm{M}$, et al. Cost-effectiveness of salmeterol xinafoate/fluticasone propionate combination inhaler in chronic asthma. Curr Med Res Opin 2007;23:1147-59.

27. Ismaila AS, Risebrough N, Li C, Corriveau D, Hawkins N, FitzGerald JM, et al. COST-effectiveness of salmeterol/fluticasone propionate combination (Advair) in uncontrolled asthma in Canada. Respir Med 2014;108:1292-302. 
28. Lundbäck B, Jenkins C, Price MJ, Thwaites RM. Cost-effectiveness of salmeterol/fluticasone propionate combination product 50/250 microg twice daily and budesonide 800 microg twice daily in the treatment of adults and adolescents with asthma. International Study Group. Respir Med 2000;94:72432.

29. Bruggenjurgen B, Ezzat N, Kardos P, Buhl R. Economic evaluation of $\mathrm{BDP} /$ formoterol fixed vs two single inhalers in asthma treatment. Allergy 2010;65:1108-15.

30. Rutten-van Molken MP, van Doorslaer EK, Till MD. Costeffectiveness analysis of formoterol versus salmeterol in patients with asthma. Pharmacoeconomics 1998;14:671-84.

31. Gerzeli S, Rognoni C, Quaglini S, Cavallo MC, Cremonesi G, Papi A. Cost-effectiveness and cost-utility of beclomethasone/formoterol versus fluticasone propionate/salmeterol in patients with moderate to severe asthma. Clin Drug Investig 2012;32:253-65.

32. Johansson G, Andreasson EB, Larsson PE, Vogelmeier CF. Cost effectiveness of budesonide/formoterol for maintenance and reliever therapy versus salmeterol/fluticasone plus salbutamol in the treatment of asthma. Pharmacoeconomics 2006;24:695-708.

33. Miller E, Sears MR, McIvor A, Liovas A. Canadian economic evaluation of budesonide-formoterol as maintenance and reliever treatment in patients with moderate to severe asthma. Can Respir J 2007;14:269-75.

34. Paggiaro P, Patel S, Nicolini G, Pradelli L, Zaniolo O, Papi A. Stepping down from high dose fluticasone/salmeterol to extrafine BDP/F in asthma is cost-effective. Respir Med 2013;107:1531-7.

35. Chongmelaxme B, Chaiyakunapruk N, Dilokthornsakul P. Incorporating adherence in cost-effectiveness analyses of asthma: a systematic review. J Med Econ 2019;22:554-66.

36. Shih Y-CT, Mauskopf J, Borker R. A cost-effectiveness analysis of first-line controller therapies for persistent asthma. Pharmacoeconomics 2007;25:577-90.

37. Zafari Z, Lynd LD, Fitzgerald JM, Sadatsafavi M. Economic and health effect of full adherence to controller therapy in adults with uncontrolled asthma: a simulation study. J Allergy Clin Immunol 2014;134:908.

38. Willson J, Bateman ED, Pavord I, Lloyd A, Krivasi T, Esser D. Cost effectiveness of tiotropium in patients with asthma poorly controlled on inhaled glucocorticosteroids and long-acting beta-agonists. Appl Health Econ Health Policy 2014;12:44759.

39. Hong SH, Cho JY, Kim TB, Lee EK, Kwon SH, Shin JY. Costeffectiveness of tiotropium in elderly patients with severe asthma using real-world data. J Allergy Clin Immunol Pract 2021;9:1939-1947.e7.

40. Zafari Z, Sadatsafavi M, Mark FitzGerald J, Canadian Respiratory Research Network. Cost-effectiveness of tiotropium versus omalizumab for uncontrolled allergic asthma in US. Cost Eff Resour Alloc 2018;16:3.

41. Menendez R, Stanford RH, Edwards L, Kalberg C, Rickard K. Cost-efficacy analysis of fluticasone propionate versus zafirlukast in patients with persistent asthma. Pharmacoeconomics 2001;19:865-74.

42. Wang L, Hollenbeak CS, Mauger DT, Zeiger RS, Paul IM, Sorkness CA, et al. Cost-effectiveness analysis of fluticasone versus montelukast in children with mild-to-moderate persistent asthma in the Pediatric Asthma Controller Trial. J Allergy Clin Immunol 2011;127:161-6.

43. Price D, Musgrave S, Wilson E, Sims E, Shepstone L, Blyth A, et al. A pragmatic single-blind randomised controlled trial and economic evaluation of the use of leukotriene receptor antago- nists in primary care at steps 2 and 3 of the national asthma guidelines (ELEVATE study). Health Technol Assess 2011;15:1-132.

44. Tan H, Sarawate C, Singer J, Elward K, Cohen RI, Smart BA, et al. Impact of asthma controller medications on clinical, economic, and patient-reported outcomes. Mayo Clin Proc 2009;84:675-84.

45. O'Connor RD, Nelson H, Borker R, Emmett A, Jhingran P, Rickard K, et al. Cost effectiveness of fluticasone propionate plus salmeterol versus fluticasone propionate plus montelukast in the treatment of persistent asthma. Pharmacoeconomics 2004;22:815-25.

46. Wilson EC, Price D, Musgrave SD, Sims EJ, Shepstone L, Murdoch J, et al. Cost effectiveness of leukotriene receptor antagonists versus long-acting beta-2 agonists as add-on therapy to inhaled corticosteroids for asthma: a pragmatic trial. Pharmacoeconomics 2010;28:597-608.

47. Borker R, Emmett A, Jhingran P, Rickard K, Dorinsky P. Determining economic feasibility of fluticasone propionatesalmeterol vs montelukast in the treatment of persistent asthma using a net benefit approach and cost-effectiveness acceptability curves. Ann Allergy Asthma Immunol 2005;95:181-9.

48. Rely K, McQuire SE, Alexandre PK, Escudero GS. Cost effectiveness of treatment with salmeterol/fluticasone compared to montelukast for the control of persistent asthma in children. Value Health 2011;14:S43-7.

49. Sheth K, Borker R, Emmett A, Rickard K, Dorinsky P. Cost effectiveness comparison of salmeterol/fluticasone propionate versus montelukast in the treatment of adults with persistent asthma. Pharmacoeconomics 2002;20:909-18.

50. Borker R, Emmett A, Jhingran P, Rickard K, Dorinsky P. Determining economic feasibility of fluticasone propionatesalmeterol vs montelukast in the treatment of persistent asthma using a net benefit approach and cost-effectiveness acceptability curves. Ann Allergy Asthma Immunol 2005;95:181-9.

51. Pathak DS, Davis EA, Stanford RH. Economic impact of asthma therapy with fluticasone propionate, montelukast, or zafirlukast in a managed care population. Pharmacotherapy 2002;22:166-74.

52. Volmer T, Effenberger T, Trautner C, Buhl R. Consequences of long-term oral corticosteroid therapy and its side-effects in severe asthma in adults: a focused review of the impact data in the literature. Eur Respir J 2018;52:1800703.

53. Bourdin A, Molinari N, Vachier I, Pahus L, Suehs C, Chanez P. Mortality: a neglected outcome in OCS-treated severe asthma. Eur Respir J 2017;50:1701486.

54. Price DB, Trudo F, Voorham J, Xu X, Kerkhof M, Ling Zhi Jie $\mathrm{J}$, et al. Adverse outcomes from initiation of systemic corticosteroids for asthma: long-term observational study. J Asthma Allergy 2018;11:193-204.

55. Sullivan PW, Ghushchyan VH, Globe G, Schatz M. Oral corticosteroid exposure and adverse effects in asthmatic patients. J Allergy Clin Immunol 2018;141:110-6.

56. Sweeney J, Patterson CC, Menzies-Gow A, Niven RM, Mansur AH, Bucknall C, et al. Comorbidity in severe asthma requiring systemic corticosteroid therapy: cross-sectional data from the Optimum Patient Care Research Database and the British Thoracic Difficult Asthma Registry. Thorax 2016;71:339-46

57. Lefebvre P, Duh MS, Lafeuille MH, Gozalo L, Desai U, Robitaille MN, et al. Burden of systemic glucocorticoid-related complications in severe asthma. Curr Med Res Opin 2017;33:57-65.

58. Barry LE, Sweeney J, O'Neill C, Price D, Heaney LG. The cost of systemic corticosteroid-induced morbidity in severe asthma: 
a health economic analysis. Respir Res 2017;18:129.

59. Canonica GW, Colombo GL, Bruno GM, Bruno GM, Di Matteo S, Martinotti C, et al. Shadow cost of oral corticosteroids-related adverse events: A pharmacoeconomic evaluation applied to real-life data from the Severe Asthma Network in Italy (SANI) registry. World Allergy Organ J 2019;12:100007.

60. Barry LE, Sweeney J, O'Neill C, Price D, Heaney LG. The cost of systemic corticosteroid-induced morbidity in severe asthma: a health economic analysis. Respir Res 2017;18:129.

61. Oba Y, Salzman G. Cost-effectiveness analysis of omalizumab in adults and adolescents with moderate-to-severe allergic asthma. J Allergy Clin Immunol 2004;114:265-9.

62. Campbell JD, Spackman DE, Sullivan SD. The costs and consequences of omalizumab in uncontrolled asthma from a USA payer perspective. Allergy 2010;65:1141-8.

63. Dal Negro RW, Tognella S, Pradelli L. A 36-month study on the cost/utility of add-on omalizumab in persistent difficult-totreat atopic asthma in Italy. J Asthma 2012;49:843-8.

64. Menzella F, Facciolongo N, Piro R, Formisano D, Roggeri A, Simonazzi A, et al. Clinical and pharmacoeconomic aspects of omalizumab: a 4-year follow-up. Ther Adv Respir Dis 2012;6:87-95.

65. Tan LE, Tan WHG, Aziz MIA, Koh MS, Tay TR, Pearce F, et al. Assessing the cost-effectiveness of mepolizumab as add-on therapy to standard of care for severe eosinophilic asthma in Singapore. J Asthma 2020;1-11. Online ahead of print.

66. Andersson M, Janson C, Kristensen T, Szende A, Golam S. Cost effectiveness of benralizumab for severe, uncontrolled oral corticosteroid-dependent asthma in Sweden. J Med Econ 2020;23:877-84.

67. Canadian Agency for Drugs and Technologies in Health. Pharmacoeconomic Review Report: Benralizumab (Fasenra): (AstraZeneca Canada Inc.). 2018. CADTH Common Drug Reviews. Accessed: 24 December 2020. Available from: https://www.ncbi.nlm.nih.gov/books/NBK541600/

68. Anderson WC 3rd, Szefler SJ. Cost-effectiveness and comparative effectiveness of biologic therapy for asthma: To biologic or not to biologic? Ann Allergy Asthma Immunol 2019;122:367-72.

69. Institute for Clinical and Economic Review, Midwest
Comparative Effectiveness Public. Biologic therapies for treatment of asthma associated with type 2 inflammation: effectiveness, value, and value-based price benchmarks: final evidence report. Boston: Institute for Clinical and Economic Review; 2018. Available from: http://resource.nlm.nih.gov/101745064

70. Shaker M, Briggs A, Dbouk A, Dutille E, Oppenheimer J, Greenhawt M. Estimation of health and economic benefits of clinic versus home administration of omalizumab and mepolizumab. J Allergy Clin Immunol Pract 2020;8:565-72.

71. Rhyou HI, Nam YH. Predictive factors of response to inhaled corticosteroids in newly diagnosed asthma: A real-world observational study. Ann Allergy Asthma Immunol 2020;125:17781.

72. Lehtimäki L, Csonka P, Mäkinen E, Isojärvi J, Hovi SL, Ahovuo-Saloranta A. Predictive value of exhaled nitric oxide in the management of asthma: a systematic review. Eur Respir J 2016;48:706-14.

73. Rodriguez-Martinez CE, Sossa-Briceño MP, Castro-Rodriguez JA. Predictors of response to medications for asthma in pediatric patients: A systematic review of the literature. Pediatr Pulmonol 2020;55:1320-31.

74. Kupczyk M, Haque S, Middelveld RJ, Dahlén B, Dahlén SE; BIOAIR Investigators. Phenotypic predictors of response to oral glucocorticosteroids in severe asthma. Respir Med 2013; 107:1521-30.

75. Gao J, Wu F. Association between fractional exhaled nitric oxide, sputum induction and peripheral blood eosinophil in uncontrolled asthma. Allergy Asthma Clin Immunol 2018;14:21.

76. Kim CH, Dilokthornsakul P, Campbell JD, van Boven JFM. Asthma cost-effectiveness analyses: Are we using the recommended outcomes in estimating value? J Allergy Clin Immunol Pract 2018;6:619-32.

77. Rodriguez-Martinez CE, Sossa-Briceño MP, Castro-Rodriguez JA. Cost effectiveness of pharmacological treatments for asthma: A systematic review. Pharmacoeconomics 2018;36:1165200.

78. Dal Negro RW, Distante C, Bonadiman L, Turco P, Iannazzo S. Cost of persistent asthma in Italy. Multidiscip Respir Med 2016;11:44.

Received for publication: 23 May 2021. Accepted for publication: 30 June 2021.

This work is licensed under a Creative Commons Attribution-NonCommercial 4.0 International License (CC BY-NC 4.0).

(C) Copyright: the Author(s), 2021

Licensee PAGEPress, Italy

Multidisciplinary Respiratory Medicine 2021; $16: 787$

doi:10.4081/mrm.2021.787 